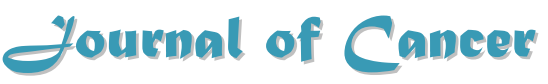

2010; 1:197-208

Research Paper

(C) Ivyspring International Publisher. All rights reserved

\title{
Ets Family Protein, Erg Expression in Developing and Adult Mouse Tissues by a Highly Specilic Monoclonal Antibody
}

\author{
Ahmed A. Mohamed¹, Shyh-Han Tan¹, Natallia Mikhalkevich1, Sathibalan Ponniah2, Valeri Vasioukhin ${ }^{3}$, \\ Charles J. Bieberich ${ }^{4}$, Isabell A. Sesterhenn ${ }^{5}$, Albert Dobi ${ }^{1}$, Shiv Srivastava ${ }^{1}$ and Taduru L. Sreenath ${ }^{1}{ }^{\otimes}$ \\ 1. Center For Prostate Disease Research, Department of Surgery, United States Military Cancer Institute, Uniformed \\ Services University of the Health Sciences, Bethesda MD 20814, USA \\ 2. Cancer Vaccine Development Laboratory, Department of Surgery, United States Military Cancer Institute, Uniformed \\ Services University of the Health Sciences, Bethesda MD 20814, USA \\ 3. Fred Hutchinson Cancer Research Center, Seattle, WA 98109, USA \\ 4. Department of Biological Sciences, University of Maryland Baltimore County, Baltimore, MD 21250, USA \\ 5. Department of Genitourinary Pathology, Armed Forces Institute of Pathology, Washington, DC, USA.
}

Corresponding author: Taduru L. Sreenath, Ph.D., Department of Surgery, Uniformed Services University. Phone: (301) 295-9821; Fax: (240) 453-8912; E-mail: tsreenath@cpdr.org

Received: 2010.10.12; Accepted: 2010.10.24; Published: 2010.10.25

\begin{abstract}
Oncogenic activation of the ETS Related Gene (ERG) in humans was originally identified in subsets of Ewing sarcomas, myeloid leukemias and, recently, in the majority of prostate cancers. Expression of human ERG protein and consequently its functions in normal and disease states needs to be better understood in light of its suggested role in cell differentiation and proliferation. Here, we analyzed temporal and spatial expression of the Erg (mouse protein) by immunohistochemical analysis during mouse embryonic and adult organogenesis using a highly specific ERG monoclonal antibody (ERG MAb). This study establishes widespread immunolocalization of Erg protein in endothelial cells and restricted expression in precartilage and hematopoietic tissues. Intriguingly, Erg is not expressed in any epithelial tissue including prostate epithelium, or in infiltrating lymphocytes that are occasionally seen in the prostate environment, a common site of tumors with ERG rearrangements and unscheduled ERG expression. These findings will further aid in investigations of Erg functions in normal and disease conditions.
\end{abstract}

Key words: Ets Related Gene, ERG, Expression, ERG MAb, Mouse, Development.

\section{Introduction}

Chromosomal translocations leading to gene $\mathrm{fu}$ sions have been well characterized in variety of malignancies [1]. Gene fusions often result in chimeric proteins with aberrant functions and/or ectopic expression. Frequent elevated expression of the ETS related genes (ERG) due to chromosomal rearrangements resulting into the fusion between androgen regulated promoters (predominatlyTMPRSS2) and protein coding sequence of ETS transcription factors has been established in prostate cancer $[2,3]$. ETS (Erythroblast Transformation Specific family of transcription factors) genes are a large family with at least thirty members that function as transcription factors [4]. All ETS transcription factors share a highly conserved DNA binding domain, the ETS domain [5] and at least Ets1, Erg, Fli1 and Etv2 are expressed in embryonic endothelial cells of mouse [6, 7]. ERG is well conserved in evolution and its expression and poten- 
tial functions have been studied in xenopus, zebrafish, mouse and humans [8-12]. The results from these studies suggest an emerging role for ERG in the transcriptional regulation of endothelial specific genes [13-16] and in definitive hematopoiesis $[17,18]$. Both hematopoietic and endothelial cells are of mesodermal origin and are derived from the hemangioblast, a common precursor, suggesting a shared developmental pathway [19]. Knock-down of Erg is associated with a significant reduction in the formation of vascular structures and the number of endothelial cells [20] and with apoptosis [21]. These studies indicate that Erg may have important implications in vascular development during mouse embryogenesis. Although Erg does not appear to be required for hematopoiesis during embryonic stem cell differentiation, it may play a role in endothelial cell differentiation [20]. Hematopoietic stem cells give rise to both $\mathrm{T}$ - and B-lymphocytes in embryogenesis and throughout adult life. Although mature T-lymphocytes do not express $E r g$, expression is detected transiently during T-lineage specification and is silenced after their commitment [22]. During B-cell development in the mouse, Erg expression was detected in early pre-B cells, pre-B and in mature B cells [23]. In developing mouse, $\operatorname{Erg}$ mRNA is expressed in mesodermal tissues such as endothelial cells, mesenchymal condensations during precartilaginous depositions, and in urogenital regions [11]. All of the expression studies were carried out by using RT-PCR or in situ hybridization. However, the protein expression and its cellular distributions could not be performed due to a lack of an Erg-specific antibody.

The goal of this study was to establish the expression pattern of Erg protein in developing and adult mouse tissues by using an ERG-specific antibody. These data would serve as a basis to understand the function of Erg during normal development in many organs and pathological conditions, such as its cancer-specific expression in prostatic adenocarcinoma. Although several antibodies for detecting human ERG protein and mouse Erg protein have been described, due to high degree of homology among ETS family members, in particular its closest homologue Fli-1, antibody cross reactivity has become a major concern in detection of the ERG protein. Recently, we have generated and characterized an ERG-specific mouse monoclonal antibody that showed high specificity towards ERG protein that does not cross react with FLI-1 protein [24]. In the present study, we examined the detailed expression of the Erg protein during prenatal and adult mouse organogenesis.

\section{Results and discussion}

Evaluation of ERG Monoclonal Antibodies for the Specificity of ERG Protein Detection: We evaluated the specificity of three recently available ERG monoclonal antibodies including the ERG MAb that we recently reported [24]. As noted previously, the ERG MAb detected ERG protein products in MOLT4, KG1, COLO 320 and VCaP tumor cell lines, whereas LNCaP, MCF7 and Jurkat cell lines were negative for ERG. The ERG MAb did not show cross reactivity to FLI-1 in LNCaP cells infected with a FLI-1 adenovirus expression vector (Fig 1A). Under similar assay conditions, rabbit monoclonal antibodies to ERG (EPR 3864 and EPR 3863) obtained from Epitomics (Burlingame, CA) detected FLI-1 in LNCaP cells infected with a FLI-1 adenovirus expression vector (Fig 1B, 1C). In addition, a rabbit monoclonal ERG antibody EPR 3864 detected a protein in Jurkat cell line (acute T cell leukemia) that was not recognized by either the rabbit monoclonal antibody EPR 3863 or our ERG $\mathrm{MAb}$ suggesting potential cross reactivity to other ETS related proteins by EPR 3864. Interestingly, other monoclonal ERG antibody EPR 3863 recognized FLI-1 in LNCaP cells infected with a FLI-1 adenovirus expression vector. Taken together, the results obtained from immunoblot analyses suggest that the ERG MAb we developed is highly specific for ERG protein detection and was further assessed in other immunoassays.

To determine the efficiency of ERG MAb antibody in a prostate tumor model, we analyzed ERG expression in ERG-positive $\mathrm{VCaP}$ and ERG-negative LNCaP prostate cancer cell tumor xenografts in SCID mice. The ERG MAb antibody detected ERG protein in $\mathrm{VCaP}$ xenografts, staining primarily the nuclei with some cytoplasmic reactivity (Fig 2A). As expected, ERG negative LNCaP xenografts did not show ERG expression. The endothelial cells lining the blood vessels and capillaries showed positive reactivity to ERG MAb in tumors as well as normal adjacent tissue (Fig 2B). Further, we evaluated the ERG MAb for the detection of ERG protein the prostates of ERG-transgenic mice [25]. Transgenic ERG expression was detected in the prostate luminal epithelial cells of ERG-transgenic mice (Fig 2C). In addition, endogenous Erg protein was detected only in the endothelial cells of blood vessels and capillaries (Fig 2D). Infiltrating lymphocytes did not show immune-reactivity to ERG MAb (Fig 2 E, G). Consistent with a recent report [26], rabbit monoclonal antibodies EPR 3864 showed a strong staining of lymphocytic infiltration in prostate gland (Fig $2 \mathrm{~F}, \mathrm{H}$ ). Interestingly, both the rabbit monoclonal ERG antibodies (EPR 3864, EPR 
3863) and FLI-1 antibodies have shown reactivity to infiltrating lymphocytes (Fig 2I, J). It is interesting to note that ERG is turned on at transition stage (between precursor and pro-T stage 1) of T-cell differentiation and does not persist stably to define T-cell identity, and is shut off after $\mathrm{T}$ cell lineage commitment $[22,27,28]$. In this study, we have not characterized the transient expression of Erg during T-cell development and differentiation.

To study the utility of the ERG MAb to detect the expression of ERG in cells using flow cytometry, seven human cancer cell lines, VCaP and LNCaP (prostate cancer), T2 and Jurkat ( $\mathrm{T}$ lymphoblastoid cells), KG-1 and KG-1a (myeloblastic) were analyzed. All cell lines were permeabilized by standard cell intracellular staining methods by using detergent followed by incubation with the ERG MAb and secondary fluorochrome-conjugated antibodies. As shown in Fig 3C and D, expression of ERG was clearly detectable in the TMPRSS2-ERG fusion-harboring VCaP cells and was not observed in the TMPRSS2-ERG negative LNCaP cells (Fig 3A, 3B). In the case of the hematopoietic/lymphoblastoid cancer cells of the T lymphocyte lineage, ERG expression was detected neither in T2 (Fig 3I, 3J) nor in Jurkat cells (Fig 3K, 3L). With the hematopoietic cell lines of myeloid lineage, KG-1 and KG-1a, the expression of ERG was clearly detectable in both cell lines (Fig 3E, $3 \mathrm{~F}$ and Fig 3G, 3H). Interestingly, the KG-1a cells, which are considered to be less mature or differentiated than the KG-1 cells, expressed much higher amounts of the ERG protein. Taken together, the consistent results of Western blot, IHC and FACS assays established the specificity of the ERG MAb in detecting ERG protein in different assay platforms and biological specimen contexts.

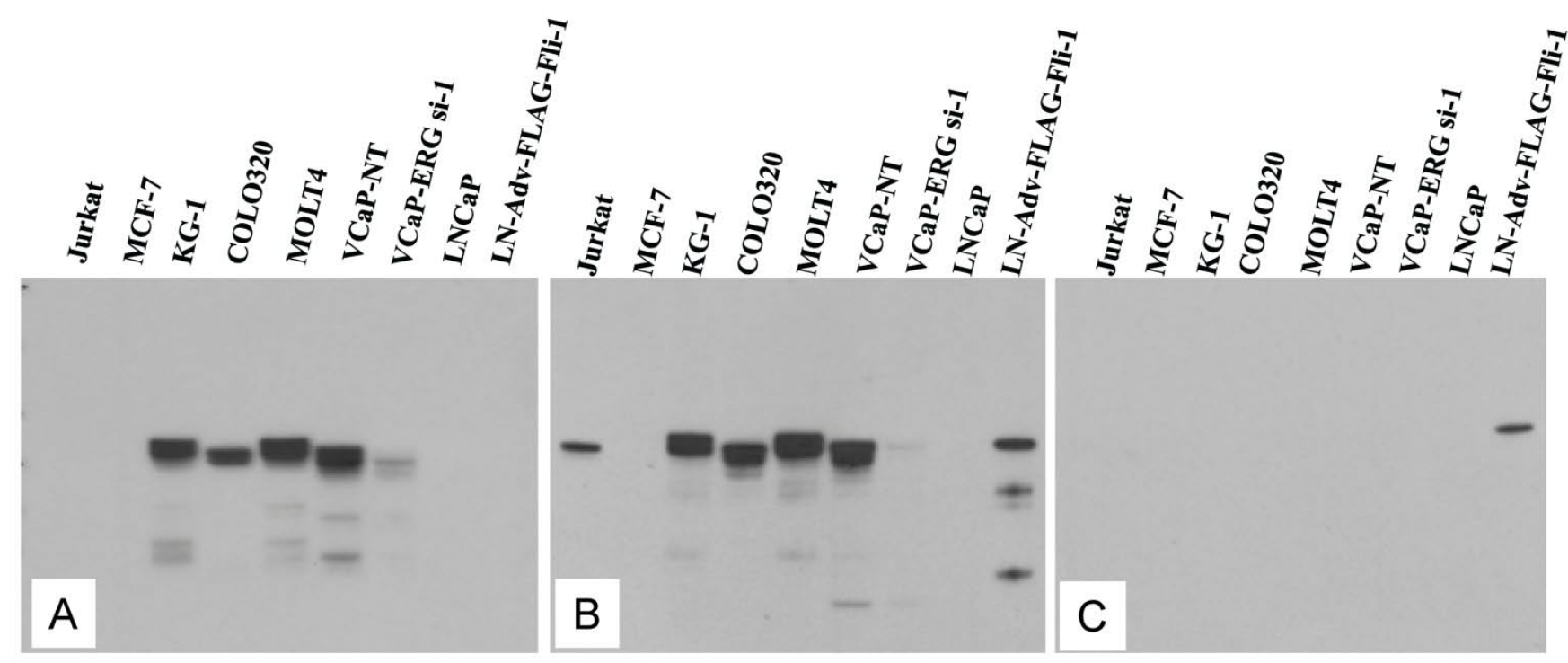

Figure I: Expression of human ERG protein in cancer-derived cell lines: ERG protein expression was analyzed by using total cell lysates from acute T cell leukemia (Jurkat), breast cancer cell line (MCF7) acute myelogenous leukemia cell line (KGI), colon carcinoma cells (COLO320), acute lymphoblastic leukemia (MOLT-4), ERG expressing prostate cancer cell line (VCaP) treated with non target siRNA (VCaP-NT), prostate cancer cell line (VCaP) treated with ERG specific siRNA (VCaP ERG-si-I), prostate cancer cell line LNCaP, prostate cancer cell line LNCaP transduced with adenoviral FLI-I expression vector. Extracted proteins were processed for immunoblot assay by using $(A)$ mouse monoclonal ERG antibodies ERG MAb, (B) rabbit monoclonal anti-ERG antibodies Epitomics EPR 3864 Cat.No 2805-I, (C). Rabbit monoclonal anti-ERG antibodies Epitomics EPR 3863 Cat.No 2849-I. Note the lack of immunoreactivity to the protein extracts from Jurkat, $\mathrm{LNCaP}$ and $\mathrm{LNCaP}$ transduced with adenoviral Fli-I expression vector with ERG MAb in panel $\mathrm{A}$, and reactivity with other antibodies in panel $\mathrm{B}$ and $\mathrm{C}$. 
Figure 2: Expression of ERG protein in FFPE tissue: ERG protein expression in (A) $\mathrm{VCaP}$ and (B) $\mathrm{LNCaP}$ xenograft tumors obtained from SCID mice. Strong expression of ERG in $\mathrm{VCaP}$ cells (black arrow). ERG staining is seen only in the endothelial cells (arrow) of LNCaP tumors but not in the epithelial cells. Expression of ERG protein is detectable in the ARR2PB $\triangle N$-ERG transgenic mouse prostates $(C)$ compared to wild-type littermate control (D). Infiltrating lymphocytes are occasionally seen in the prostate glands and show no reactivity to ERG MAb $(E, G)$. However, strong staining is seen in the infiltrating lymphocytes with Epitomics EPR $3864(\mathrm{~F}, \mathrm{H})$, EPR 3863 (I) and FLII antibody (J).

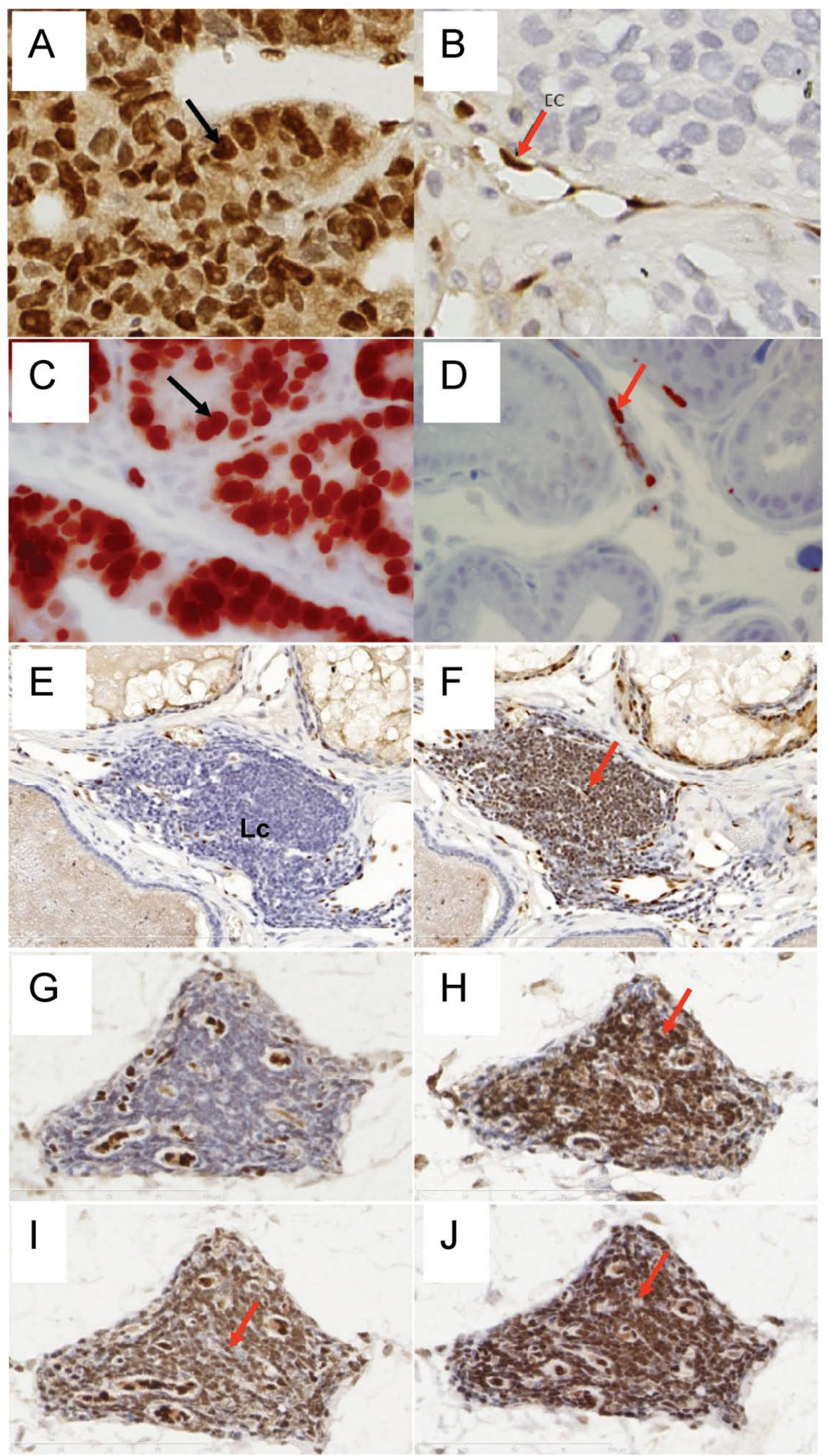


Figure 3. ERG expression in cancer cell lines. Intracellular staining was carried out in permeabilized cells with an IgGI isotype control antibody (A, C, E, G, I and K) or for ERG with ERG MAb (B, D, F, H, J and $L)$ and detected by using FITC-conjugated secondary antibody and flow cytometry analysis.

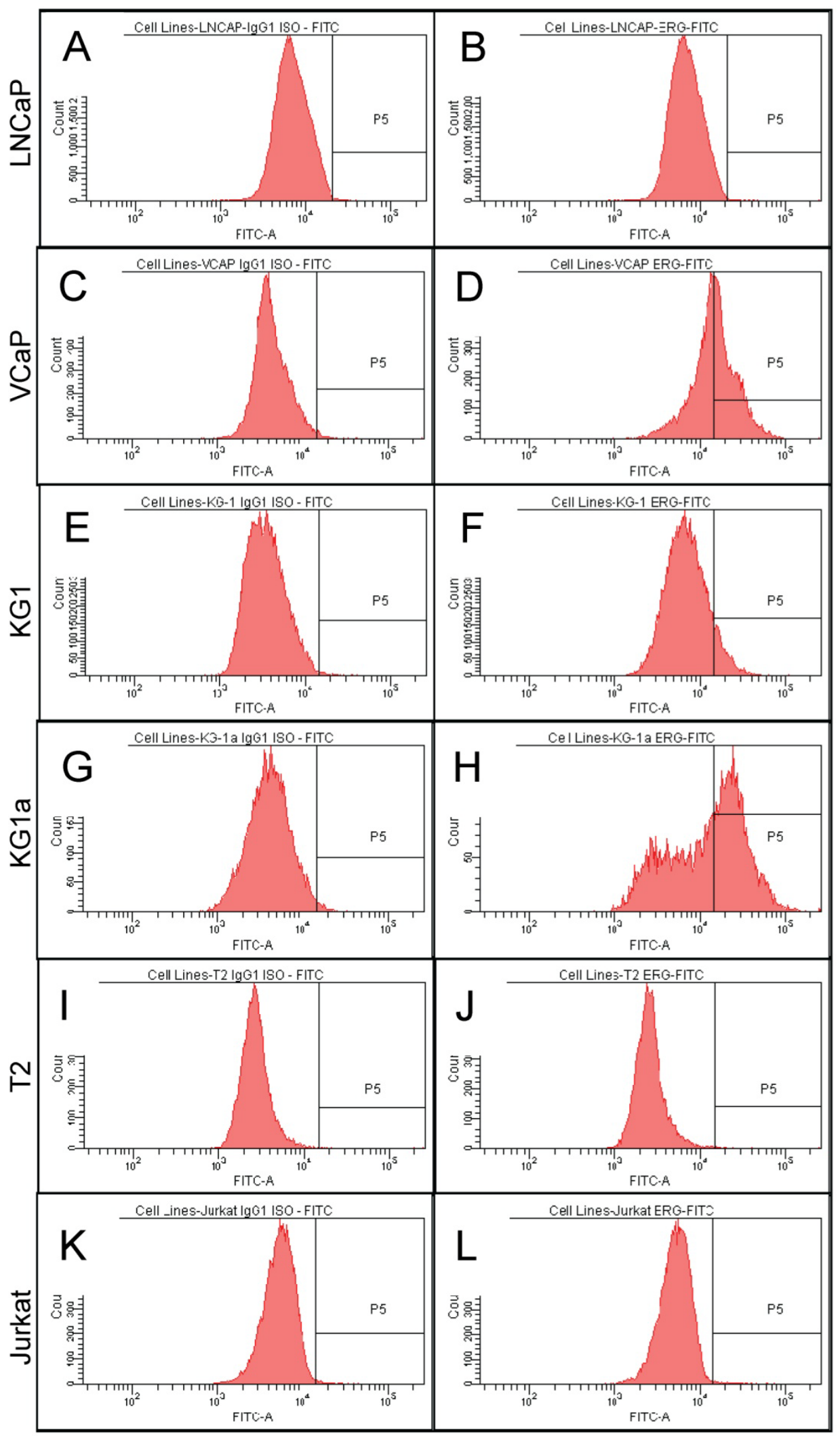

Isotype control

ERG MAb 
Erg protein expression in prenatal mouse development: During E9.5d, strong expression of Erg protein was detected by the ERG MAb mainly in the endothelial cells of blood vessels around the neural tube (Fig 4A, 4B). Additionally, expression was also prominent in the cells that line the amnion (Fig 4A). At this stage, during heart development, the ventricle begins trabeculation to demarcate this region from the primitive heart. Expression of Erg was observed in endothelial cells that line the trabeculated regions of the ventricle (Fig 4C). Endothelial cells present in the inter-somitic capillaries show strong expression of Erg (Fig 4D). Similar endothelial-specific expression was found in the dorsal aorta and around the neural tube (Fig 4E). The distribution observed with ERG MAb antibody is consistent with earlier reported studies of Erg mRNA expression [11, 20]. Similar to earlier stages of development, at E12.5d, Erg expression was endothelial cell-specific in the majority of the tissues (Fig 5). In addition to endothelial expression, Erg expression was detected in the precartilage/ cartilage primordium of the nasal septum, neural arch and rib
(Fig 5A, 5B, 5C). Mesenchymal condensations are required at this stage to initiate the paving cartilage path for both transient and permanent cartilage. The transient cartilage will undergo ossification to form bone. Interestingly, Erg expression was observed only in the precartilage primordium suggesting that Erg may have critical role in the differentiation of cartilage. Heart development at this stage exhibited extensive trabeculation of the ventricle and showed clear lining of endothelial cells with positive Erg staining along the trabeculated endocardium (Fig 5B). Lungs at this stage were not yet divided into lobes and the stroma with enriched capillaries exhibited strong expression of Erg in developing lung (Fig 5D). Epithelial cells of segmental bronchus did not show Erg expression (Fig 5D). Kidney at this stage starts subdividing into cortical and medullary regions. Expression was detected only in the blood vessels and capillaries uniformly throughout the kidney and not in the kidney cortex or medulla (Fig 5E).
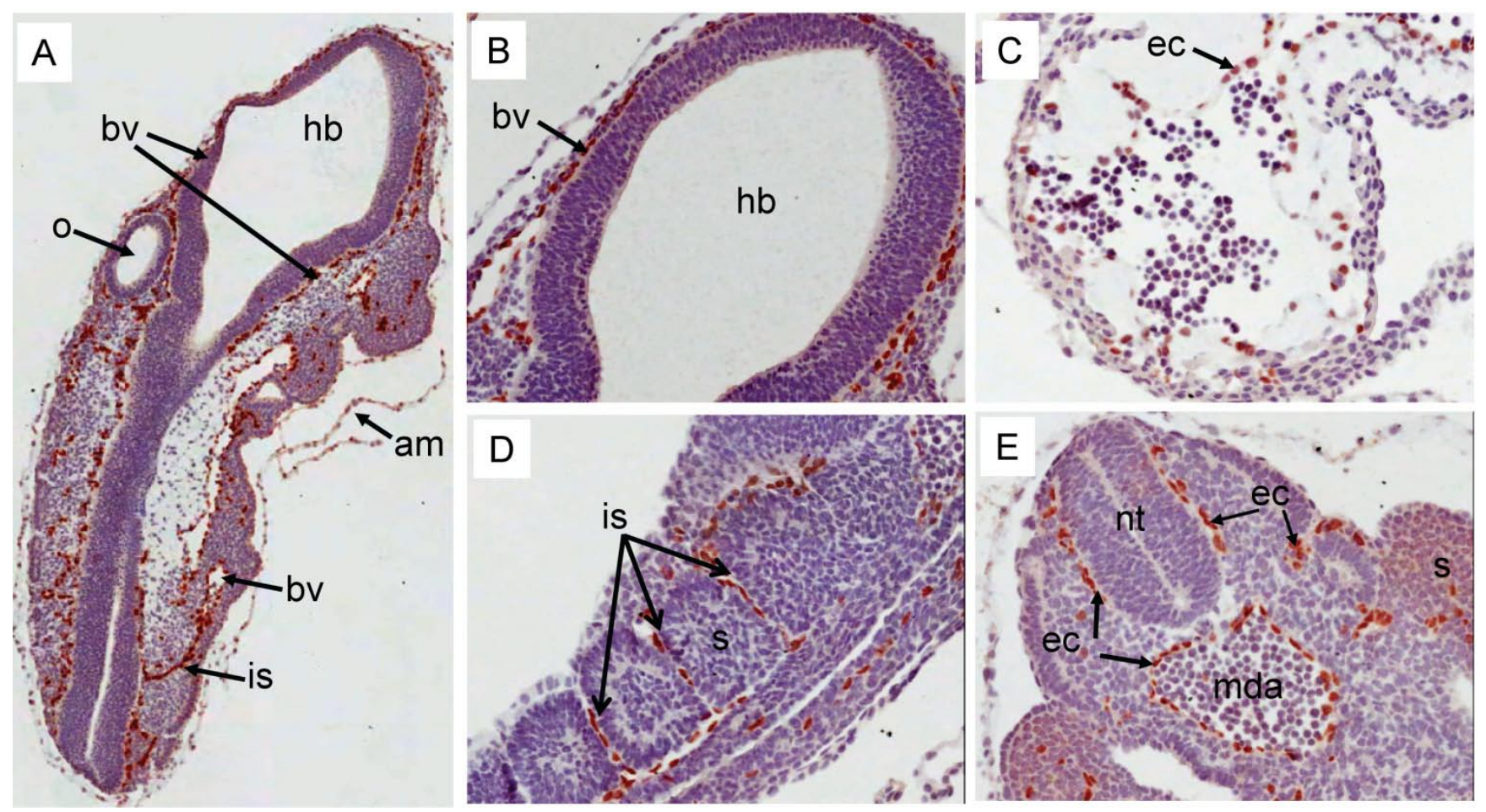

Figure 4. Expression pattern of Erg protein during mouse embryogenesis (E9.5d): Embryonic $9.5 \mathrm{~d}$ mouse showing the expression of Erg protein by immunohistochemistry with ERG MAb. (A) Coronal section of an E9.5 embryo showing a specific staining in blood vessels (bv), inter-somitic vessels (is) and in the amnion (am). (B) Higher magnification of hind brain. Expression is not seen in the hind brain (hb), neural tube (nt) and optic vesicle (o). (C). Higher magnification of ventricle (vt) region of the heart showing strong signal in the endothelial cells (ec)along the trabeculated endochordium (D) Hihger magnification of somites in the caudal region showing Erg expression in the inter somatic blood vessels (sv) . (E). Tail region of the embryo showing neural tube (nt) midline dorsal aorta (mda). Erg expression was detectable only in the endothelial cells of dorsal aorta. Somites (s). 

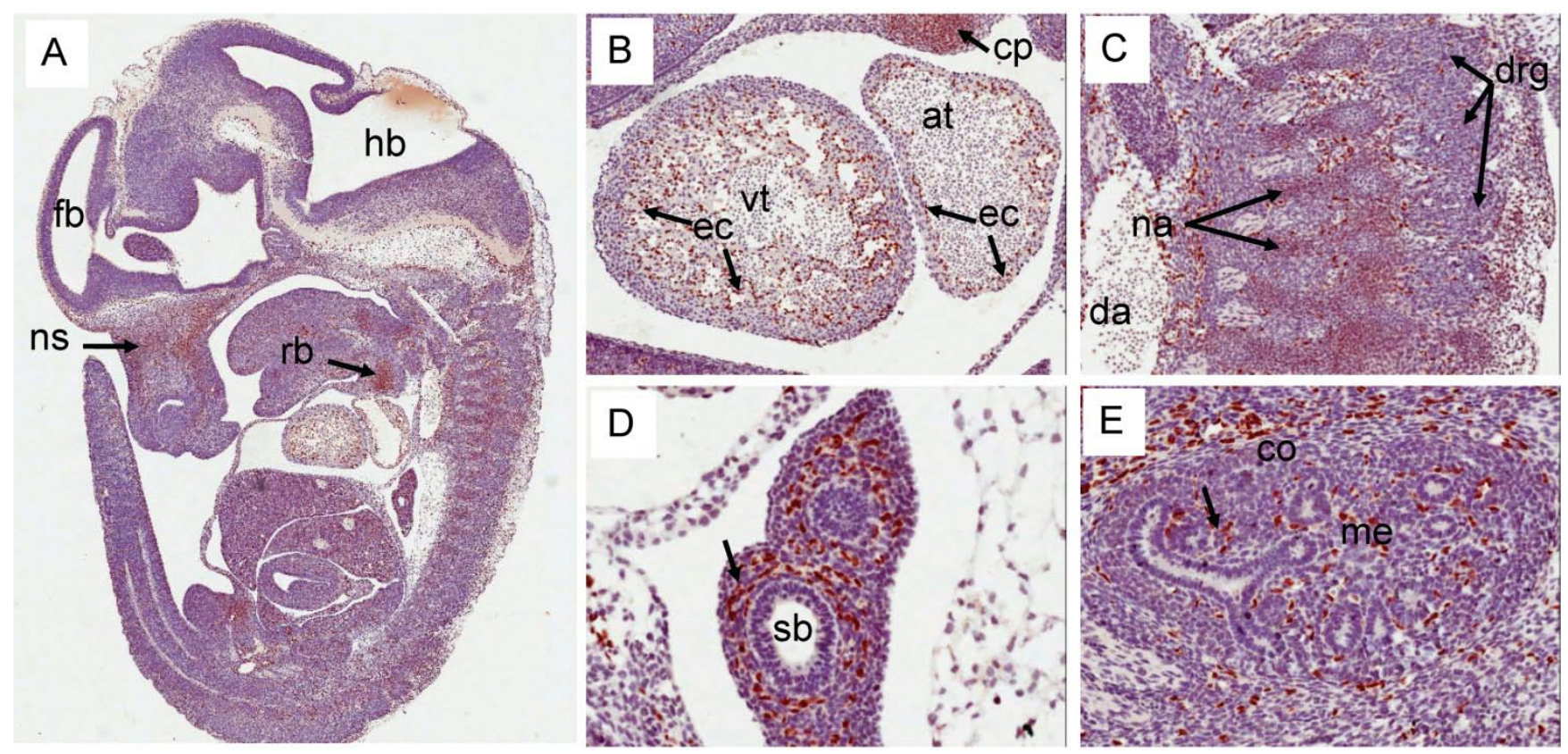

Figure 5. Expression pattern of Erg protein during mouse embryogenesis: (EI2.5). (A) Sagittal section of an EI2.5d embryo showing a specific staining in cartillage primordium (cP) of the nasal septum (ns), and the mid shaft region of the rib (rb). (B) Higher magnification of ventricle (vt) region of the heart showing strong expression in the endothelial cells (ec) along the trabeculated endocordium. (C) Erg protein was detectable in the precartillage condensations in the neural arch (na). (D) Higher magnification of developing lungs (not yet divided into lobes) show lack of expression in the epithelial cells of segmental bronchus (sb). Surrounding stroma with enrihed capillaires exhibit strong staining. (E). Expression is seen only in endothelial cells of the blood vessels and capillaries uniform throughout the kidney.

Erg expression in E14.5d was found mostly in the endothelial cells of variety of tissues (Fig 6). In developing liver, about 1-2\% of liver cells exhibited reactivity with ERG MAb antibody and megakaryocytes did not show Erg expression. Expression in the cartilage appeared to be reduced significantly compared to E12.5d in the rib, nasal septum and vertebrae. We found significant differences between ERG $\mathrm{MAb}$ and rabbit monoclonal ERG antibodies EPR 3864 megakaryocytic immunostaining. No significant changes in the Erg expression patterns were observed in the later stages of mouse development. As the overexpression of ERG in the prostate is implicated in the oncogenic process, we examined the normal expression of Erg protein in developing prostate glands. In the mouse, the prostatic buds first emerge at the rostral end of the urogenital sinus at approximately 17.5 days of gestation and subsequently, the prostatic epithelial buds undergo extensive ductal outgrowth and branching morphogenesis into the surrounding mesenchyme during the first three weeks of postnatal development. Interestingly, Erg staining was observed only in the mesenchymal compartment and restricted to capillary endothelial cells (Fig 7) suggesting that Erg may not be critical in normal prostate development or differentiation.
In liver of E17.5d mice, the expression of Erg is restricted to very few cells such as endothelial and other non-parenchymal cells which later differentiate into Kupffer cells and hepatic stellate cells. It is not clear at this stage which type of cells in addition to endothelial cells express Erg protein. Similar to E14.5d liver megakaryocytes, ERG MAb did not show reactivity with E17.5d hepatic megakaryocytes (Fig 8A). However, rabbit monoclonal ERG antibodies EPR 3864 showed a strong staining in megakaryocytes, in addition to endothelial and other cell types (Fig 8B).

Expression of Erg protein in adult mice: We extended our study to adult mouse tissues to analyze the expression of Erg protein (Fig 9). Similar to embryonic tissues, the expression of Erg was observed in the endothelial cells of the adrenal gland, cartilaginous component of bone, heart muscle, kidney, liver, lung, spleen urinary bladder. Erg expression was also evident in the lymphatic endothelial cells in adult mouse. As expected, expression was undetectable in the prostate epithelium. Detailed examination of these tissues has revealed that the expression was mostly restricted to hematopoietic and endothelial compartments. In liver, the expression appeared to be in the Kupffer cells. In bone marrow, ERG MAb did not show reactivity with megakaryocytes. Interestingly, 
eosinophils, with a characteristic ring shaped or segmented/multilobed nuclei stained the nuclei with ERG MAb. Eosinophils are derived from hematopoietic stem cells initially committed to the myeloid line and then to the basophil-eosinophil granulocyte lineage. The presence of Erg in these cells suggest its potential function in either differentiation or maintenance of differentiation state. During development, Fli1 is preferentially expressed in hematopoietic cells, endothelial cells and in the mesenchyme which is mainly derived from neural crest cells [29]. Similar to earlier observations [11] we also observe the co-expression of the Erg and Fli1 genes in both endothelial and mesodermal tissues, including urogenital tract and precartilaginous areas. Similar to Erg, Fli1 expression also appeared transient during mouse embryogenesis. This result is consistent with subtle temporal regulation. Whether this spatiotemporal overlap determines redundant functions or not remains to be determined. Due to specificity and clarity of Erg detection in developing and adult mouse tissues, these findings will be valuable in further assessing in vivo functions of Erg in normal and malignant tissues.
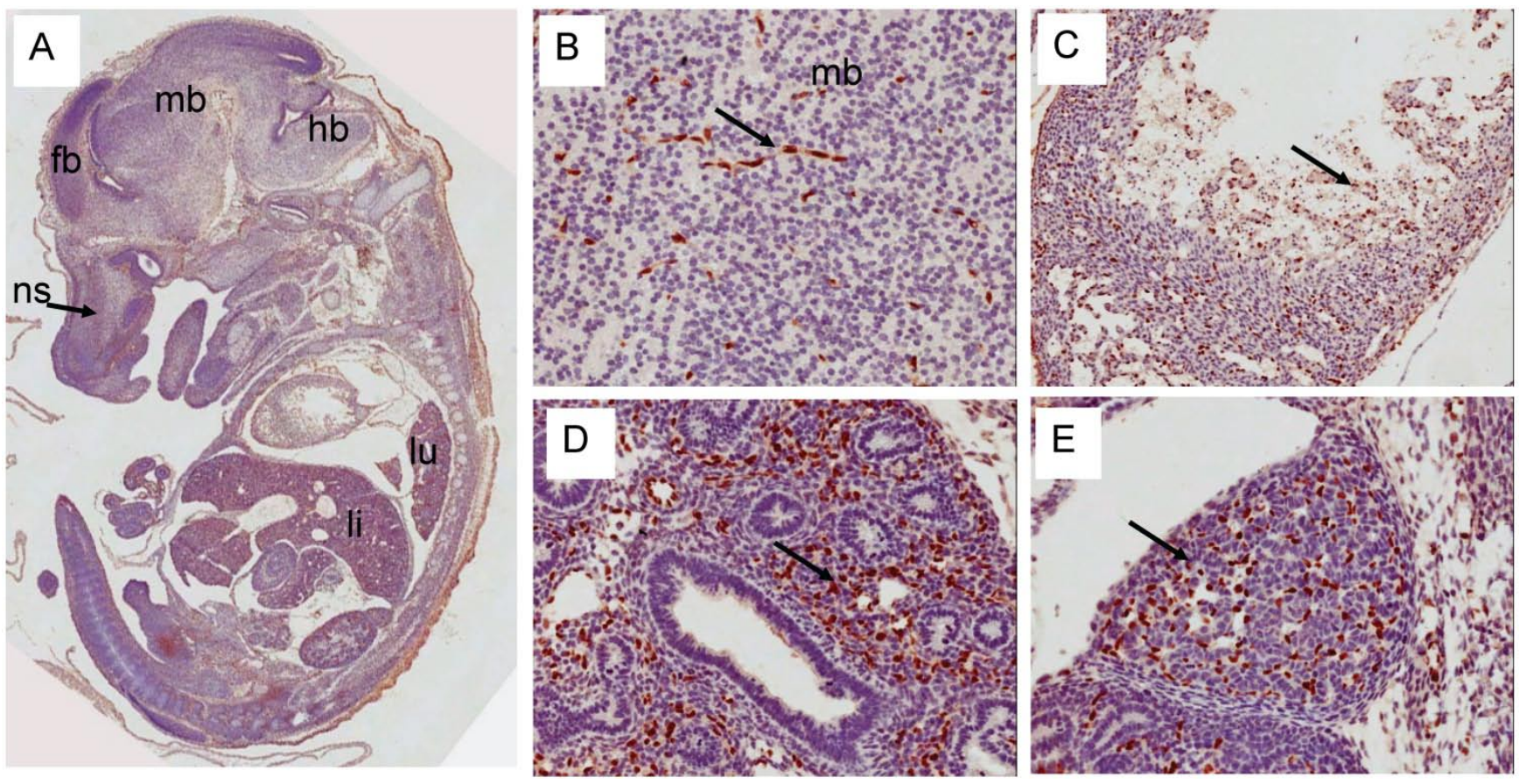

Figure 6. Expression pattern of Erg protein during mouse embryogenesis (EI4.5): (A) Sagittal section of an EI4.5 embryo showing a specific staining in cartillage primordium (cP) of the nasal septum (ns) similar to EI2.5d. Higher magnification of midbrain showing capillaries staining of Erg. (C) Higher magnification of ventricle (vt) region of the heart showing strong ractivity in the endothelial cells (ec) along the trabeculated endocordium. (D) Higher magnification of developing lungs shows lack of expression in the epithelial cells of segmental bronchus (sb). Stroma with enrihed capillaires exhibit strong staining. (E). Higher magnification of adrenal gland with endothelial cell specific Erg staining. 

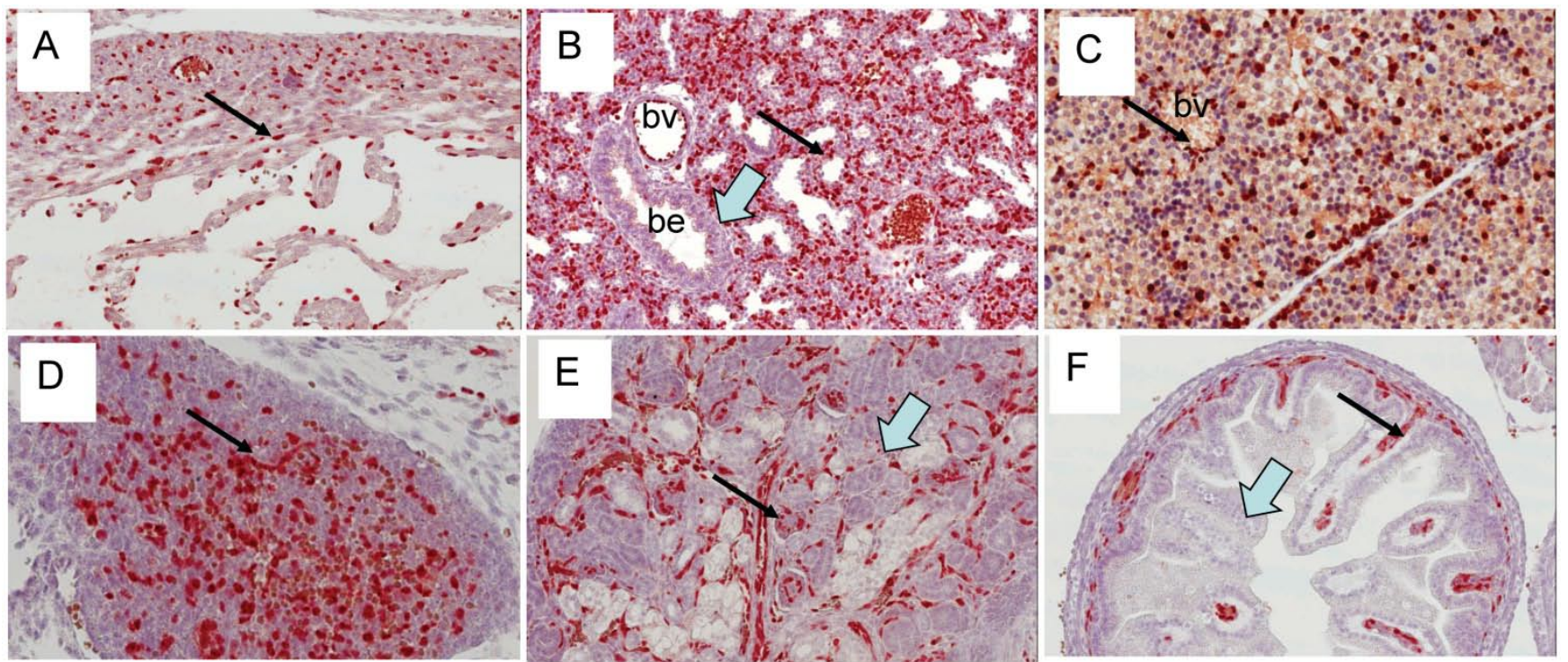

Figure 7. Expression pattern of Erg protein during mouse embryogenesis(EI7.5): (A) Ventricle showing trebeculated endocordium with endothelial specific staining of Erg. (B). High magnification of lung showing capillaries and blood vessel specific Erg reactivity. Note the lack of staining in the lung epithelial. (C) High magnification of liver showing a specific staining is non-hepatocytes in addition to endothelial cells. (D) High magnification of adrenal gland showing random Erg in both cortex and medullary region consistent with fenestrated vessels. Similarly, kidney (E) and intestine (F) show endothelial specific staining, Open arrows show the lack of expression in the bronchial epithelial cells (B), kidney tubules (E) and intestinal epithelial cells in the cripte (F). Blood vessel (bv), bronchial epithelium (be).

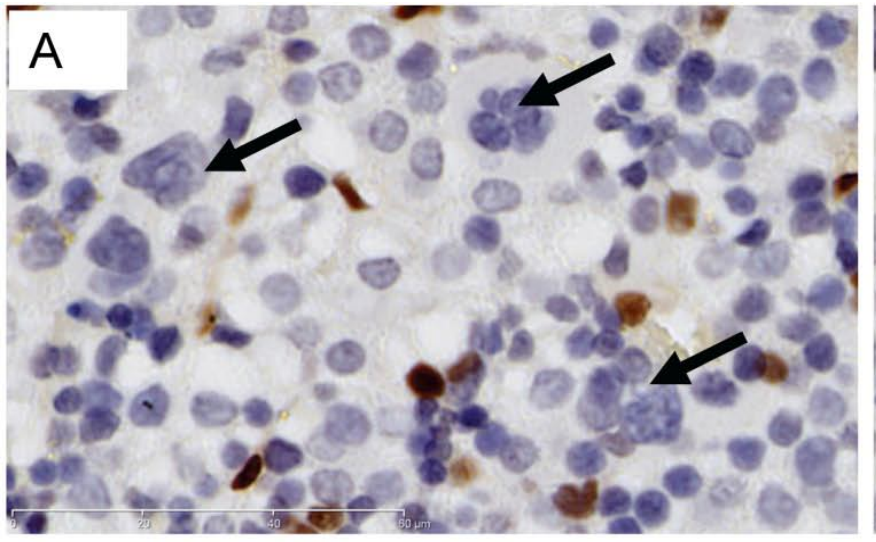

ERG MAb

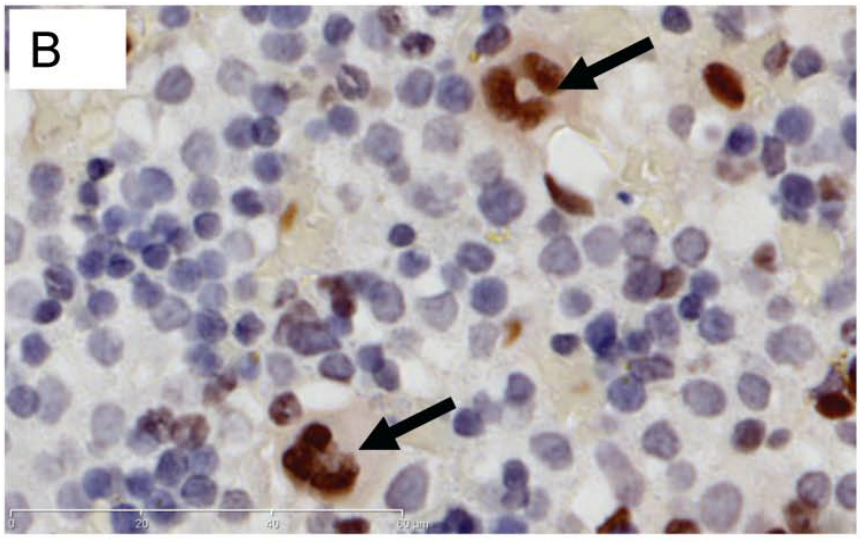

ERG (EPR 3864 ) Epitomics

Figure 8. Expression of Erg protein in developing liver (EI7.5). (A) Detection of Erg protein in endothelial and non-paranchymal cells by ERG MAb. The arrows point to megakaryocytes that lack the expression of Erg protein. (B). Erg staining with rabbit monoclonal ERG antibodies Epitomics EPR 3864 show endothelial and non-paranchymal cells staining. Megakaryocytes know to have expression of Flil also are detected with EPR 3864. Similar megakaryocyte specific expression is also observed with EPR 3863 and FLII antibodies (data not shown). 


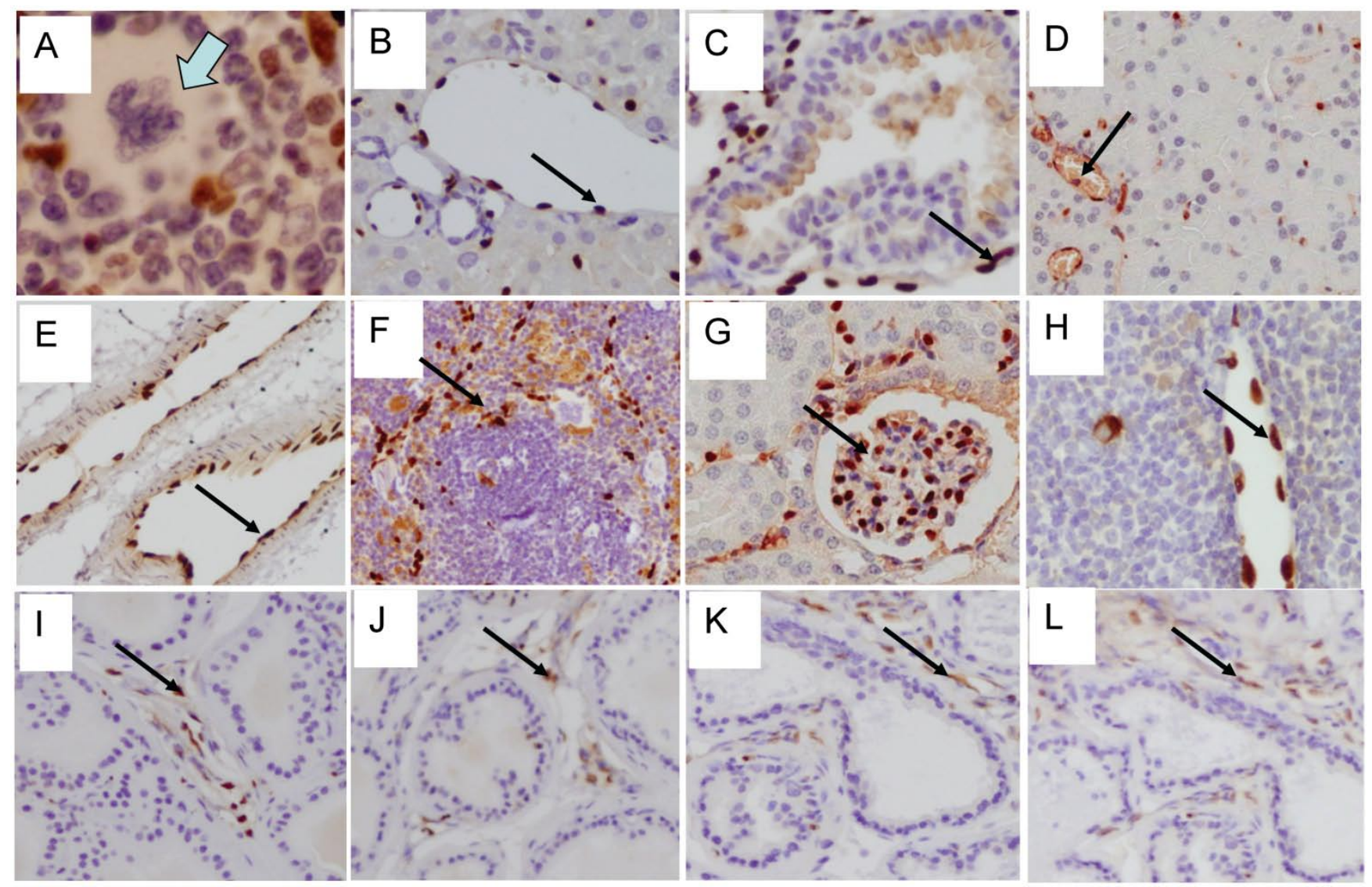

Figure 9. Expression of Erg protein in adult tissues: (A) Bone marrow (B) Liver (C) Lung (D) Pancreas (E) Lymphatic vessels (F) Spleen (G) Kidney (Glomeruli) $(\mathrm{H})$ Thymus (I) Anterior prostate (J) Dorsal prostate (K) Lateral prostate (L) Ventral prostate. Expression is seen mainly in the endothelial cells in blood vessels and capillaries (arrows). In bone marrow, megakaryocyte show lack of Erg expression (open arrow).

\section{Materials and Methods}

Antibodies: Recently, we have reported the generation and characterization of mouse monoclonal antibodies to ERG, showing higher specificity [24]. In this study, we have used mouse monoclonal ERG antibodies along with the other commercially available ERG antibodies, a rabbit anti-ERG monoclonal antibody clone EPR 3864 (Cat No. 2805-1) [26] and clone EPR 3863 (Cat No. 2849-1) obtained from Epitomics, Burlingame, CA and FLI-1 antibodies from Dr. Denis Watson, University of South Carolina, Charleston, SC. Anti-GAPDH (sc-25778, Santa Cruz Biotechnology, Santa Cruz, CA) sheep anti-mouse IgG-HRP (NXA931, GE Health Care, Buckinghamshire, UK) donkey anti-rabbit IgG-HRP (NA934, GE Health Care, Buckinghamshire, UK)

Western blot assays. Cells (Jurkat, MCF7, KG1, Molt4, VCaP, LNCaP) were lysed in Mammalian Protein Extraction Reagent (M-PER) (Pierce, Rockford, IL) containing protease inhibitor cocktail and phosphatase inhibitor cocktails I \& II (Sigma, St Louis,
$\mathrm{MO})$. Cell lysates equivalent to $50 \mu \mathrm{g}$ of protein were separated on 4-12\% Bis-Tris Gel (Invitrogen, Carlsbad, $\mathrm{CA}$ ) and transferred to PVDF membrane (Invitrogen, Carlsbad, CA). Membranes were incubated with primary antibodies: Anti-ERG mouse monoclonal antibody (ERG MAb)[24] (1:500 dilution), rabbit monoclonal ERG (EPR 3864 and EPR 3863) antibodies (1:500 dilution) anti-GAPDH (sc-25778, Santa Cruz Biotechnology, Santa Cruz, CA) at $4{ }^{\circ} \mathrm{C}$ for overnight. Membranes were washed three times for 5 minutes each at room temperature followed by treatment with secondary antibodies: sheep anti-mouse IgG-HRP or donkey anti-rabbit IgG-HRP at $24^{\circ} \mathrm{C}$ for 1 hour. Finally membranes were washed three times and bands were visualized with ECL Western blot detection reagent (GE Health Care, Buckinghamshire, UK).

Immunofluorescence staining for ERG in cell lines and flow cytometry analysis. The cancer cell lines used in this study were obtained from ATCC. $\mathrm{VCaP}$ cells were grown in DMEM/10\%FCS+Penicillin/Streptomycin/L-Glutam ine. LNCaP, T2, Jurkat, KG-1 and KG-1a cells were 
cultured in RPMI/10\%FCS/Penicillin/ Streptomycin/L-Glutamine. On the day of the assay adherent cell lines ( $\mathrm{VCaP}, \mathrm{LNCaP})$ were trypsinized to yield single cell suspensions while the non-adherent cells (T2, Jurkat, KG-1 and KG-1a) were utilized after a washing step. For each cell line, two aliquots of $5 \times 10^{5}$ cells/tube were permeabilized with freshly prepared permeabilization buffer as directed by the manufacturer (eBioscience). One aliquot was stained with an IgG1 isotype control antibody (Invitrogen) and the second aliquot was stained with the anti-ERG antibody at a 1:20 dilution for 1 hour at $4^{\circ} \mathrm{C}$. Then cells were washed and stained with FITC-conjugated rat anti-mouse IgG1 antibody (Clone A85-1, BD Pharmingen) for an additional 30 minutes at $4^{\circ} \mathrm{C}$. Cells were then washed and analyzed by using a BD FACS Canto II flow cytometer. Data was collected on the total cell population and the analysis was performed by using FACS Diva software (version 5 .03) (Becton Dickinson).

Tissues and processing for Immunohistochemistry: Fixation and impregnation FVB/N and C57BL6 mice embryos from various stages of development were dissected from pregnant females, washed in ice-cold phosphate-buffered saline (PBS) and fixed in ice-cold $4 \%$ paraformaldehyde overnight, sequentially dehydration, embedding, and sectioning steps were performed according to established protocols.

Preparation for staining: Slides (with 5-mm sections) were warmed at $60^{\circ} \mathrm{C}$ for 15 minutes and immediately immersed in xylene for effective removal of paraffin and processed additionally twice in xylene for $5 \mathrm{~min}$ each, washed twice in $100 \%$ ethanol for 5 min each, and subsequently rehydrated in $95,75 \%$ ethanol, and PBS for $5 \mathrm{~min}$ at room temperature.

Immunohistochemistry: Antigen retrieval was performed on these slides in Antigen unmasking solution (Vector Biolabs, Burlingame, CA) by using vegetable steamer for 45 minutes and the slides were allowed to cool to room temperature for about $30 \mathrm{~min}$. Slides were washed twice in 1xPBS and treated with $3 \% \mathrm{H}_{2} \mathrm{O}_{2}$ in ultrapure water for 15 minutes to quench the endogenous peroxidase activity. Immunodetection was performed using Mouse-to-mouse detection system kit according manufacturer's instructions (Millipore Inc. Billerica, MA). Briefly, nonspecific binding of the antibody was blocked by incubating the slides with pre-blocking solution for 10 minutes at RT, primary antibodies $(200 \mathrm{pg} / \mathrm{ml}$ in $10 \%$ normal goat serum) at $4{ }^{\circ} \mathrm{C}$ overnight or room temperature for 2 hours. Slides were washed twice in 1xPBS sites 5 min each, incubated with post-antibody blocking solution for $10 \mathrm{~min}$. Slides were washed twice in 1xPBS sites $5 \mathrm{~min}$ each and incubated with ready-to-use poly-HRP-Anti-Mouse/Rabbit IgG for 30 minutes, washed twice with 1xPBS for 5 minutes each. Color development was performed by using $\mathrm{DAB}$ as a substrate for peroxidase enzyme activity. The color reaction was stopped by washing/rinsing slides in tap water several times. Slides were counterstained with Hematoxylene for 2 minutes, rinsed in tap water for 5 mins, dehydrated sequentially in ascending concentration of alcohol, cleared in xylene and permanently mounted with Permont solution. The slides were scanned in Biomedical Instrumentation Center at Uniformed Services University of the Health Sciences using Olympus NanoZoomer Digital Pathology microscope at 40X magnification and digital images were taken from the scans.

\section{Acknowledgements}

Authors wish to express sincere thanks to Dr David G. McLeod, Dr. David Burris and Dr. Norman Rich for their continued support throughout the work. The authors are grateful to Dr. Gyorgy Petrovics, CPDR/USU, Dr. Alfredo Molinolo, NIDCR/NIH for their valuable advices and comments during the progress of this study, Mr. Mohamed Mursal for technical assistance in FACS analysis. This work was supported by the CPDR Program HU0001-04C-1502 to David G. McLeod., NIH grant 1RO1 DK065977 to S.S and DoD grant PC073614 to T.S., A.D and S.S. The views expressed in this manuscript are those of the authors and do not reflect the official policy of the Department of the Army, Department of Defense or the U.S. Government.

\section{Conflict of Interest}

The authors do not have any conflict of Interest.

\section{References}

1. Aplan PD. Causes of oncogenic chromosomal translocation. Trends Genet, 2006. 22(1): 46-55.

2. Demichelis F, Fall K, Perner S, et al. TMPRSS2:ERG gene fusion associated with lethal prostate cancer in a watchful waiting cohort. Oncogene, 2007. 26(31): 4596-9.

3. Tomlins SA, Laxman B, Dhanasekaran SM, et al. Distinct classes of chromosomal rearrangements create oncogenic ETS gene fusions in prostate cancer. Nature, 2007. 448(7153): 595-9.

4. Rao VN, Papas TS, and Reddy ES. erg, a human ets-related gene on chromosome 21: alternative splicing, polyadenylation, and translation. Science, 1987. 237(4815): 635-9.

5. Graves, BJ and Petersen JM. Specificity within the ets family of transcription factors. Adv Cancer Res, 1998. 75: 1-55.

6. Lee D, Park C, Lee H, et al. ER71 acts downstream of BMP, Notch, and Wnt signaling in blood and vessel progenitor specification. Cell Stem Cell, 2008. 2(5): 497-507.

7. Lelievre E, Lionneton F, Soncin F, and Vandenbunder B. The Ets family contains transcriptional activators and repressors 
involved in angiogenesis. Int J Biochem Cell Biol, 2001. 33(4): 391-407.

8. Dhordain P, Dewitte F, Desbiens X, Stehelin D, and Duterque-Coquillaud M. Mesodermal expression of the chicken erg gene associated with precartilaginous condensation and cartilage differentiation. Mech Dev, 1995. 50(1): 17-28.

9. Ganan Y, Macias D, Duterque-Coquillaud M, Ros MA, and Hurle JM. Role of TGF beta $\mathrm{s}$ and BMPs as signals controlling the position of the digits and the areas of interdigital cell death in the developing chick limb autopod. Development, 1996. 122(8): 2349-57.

10. Baltzinger M, Mager-Heckel AM, and Remy P. Xl erg: expression pattern and overexpression during development plead for a role in endothelial cell differentiation. Dev Dyn, 1999. 216(4-5): 420-33.

11. Vlaeminck-Guillem V, Carrere S, Dewitte F, Stehelin D, Desbiens $\mathrm{X}$, and Duterque-Coquillaud $\mathrm{M}$. The Ets family member Erg gene is expressed in mesodermal tissues and neural crests at fundamental steps during mouse embryogenesis. Mech Dev, 2000. 91(1-2): 331-5.

12. Pham VN, Lawson ND, Mugford JW, et al. Combinatorial function of ETS transcription factors in the developing vasculature. Dev Biol, 2007. 303(2): 772-83.

13. Schwachtgen JL, Janel N, Barek L, et al. Ets transcription factors bind and transactivate the core promoter of the von Willebrand factor gene. Oncogene, 1997. 15(25): 3091-102.

14. Gory S, Dalmon J, Prandini MH, Kortulewski T, de Launoit $Y$, and Huber P. Requirement of a GT box (Sp1 site) and two Ets binding sites for vascular endothelial cadherin gene transcription. J Biol Chem, 1998. 273(12): 6750-5.

15. Hasegawa $Y, A$ be $M$, Yamazaki $T$, et al. Transcriptional regulation of human angiopoietin-2 by transcription factor Ets-1. Biochem Biophys Res Commun, 2004. 316(1): 52-8.

16. Pimanda JE, Chan WY, Donaldson IJ, Bowen M, Green AR, and Gottgens B. Endoglin expression in the endothelium is regulated by Fli-1, Erg, and Elf-1 acting on the promoter and a $-8-\mathrm{kb}$ enhancer. Blood, 2006. 107(12): 4737-45.

17. Loughran SJ, Kruse EA, Hacking DF, et al. The transcription factor Erg is essential for definitive hematopoiesis and the function of adult hematopoietic stem cells. Nat Immunol, 2008. 9(7): 810-9.

18. Kruse EA, Loughran SJ, Baldwin TM, et al. Dual requirement for the ETS transcription factors Fli-1 and Erg in hematopoietic stem cells and the megakaryocyte lineage. Proc Natl Acad Sci U S A, 2009. 106(33): 13814-9.

19. Jaffredo T, Nottingham W, Liddiard K, Bollerot K, Pouget C, and de Bruijn M. From hemangioblast to hematopoietic stem cell: an endothelial connection? Exp Hematol, 2005. 33(9): 1029-40.

20. Nikolova-Krstevski V, Yuan L, Le Bras A, et al. ERG is required for the differentiation of embryonic stem cells along the endothelial lineage. BMC Dev Biol, 2009. 9: 72.

21. Birdsey GM, Dryden NH, Amsellem V, et al. Transcription factor Erg regulates angiogenesis and endothelial apoptosis through VE-cadherin. Blood, 2008. 111(7): 3498-506.

22. Anderson MK, Hernandez-Hoyos G, Diamond RA, and Rothenberg EV. Precise developmental regulation of Ets family transcription factors during specification and commitment to the T cell lineage. Development, 1999. 126(14): 3131-48.

23. Rivera RR, Stuiver MH, Steenbergen R, and Murre C. Ets proteins: new factors that regulate immunoglobulin heavy-chain gene expression. Mol Cell Biol, 1993. 13(11): 7163-9.

24. Furusato B, Tan SH, Young D, et al. ERG oncoprotein expression in prostate cancer: clonal progression of ERG-positive tumor cells and potential for ERG-based stratification. Prostate Cancer Prostatic Dis, 2010. 13(3): 228-37.
25. Klezovitch O, Risk M, Coleman I, et al. A causal role for ERG in neoplastic transformation of prostate epithelium. Proc Natl Acad Sci U S A, 2008. 105(6): 2105-10.

26. Park K, Tomlins SA, Mudaliar KM, et al. Antibody-based detection of ERG rearrangement-positive prostate cancer. Neoplasia, 2010. 12(7): 590-8.

27. Rothenberg EV, Telfer JC, and Anderson MK. Transcriptional regulation of lymphocyte lineage commitment. Bioessays, 1999. 21(9): 726-42.

28. Rothenberg EV. Stepwise specification of lymphocyte developmental lineages. Curr Opin Genet Dev, 2000. 10(4): 370-9.

29. Melet F, Motro B, Rossi DJ, Zhang L, and Bernstein A. Generation of a novel Fli-1 protein by gene targeting leads to a defect in thymus development and a delay in Friend virus-induced erythroleukemia. Mol Cell Biol, 1996. 16(6): 2708-18. 